\title{
Representationalism and the Problem of Vagueness
}

RYAN PERKINS \& TIM BAYNE

Comments welcome: m.ryan.perkins@gmail.com

This paper develops a novel problem for representationalism, a popular contemporary account of perception. To a first approximation, representationalism holds that there is a close connection between phenomenal consciousness and perceptual representation. The nature of the relevant connection is discussed below. We shall argue that representationalism is incompatible with the conjunction of two attractive claims:
A. Perceptual representation is vague.
B. Phenomenal consciousness is precise.

As it stands, (A) and (B) are mere slogans; their contents are unpacked in a rigorous manner later on. Nevertheless, even in their inchoate present condition, the potential for incompatibility with representationalism should be clear: representationalism holds that there is an intimate connection between phenomenal consciousness and perceptual representation; (A) and (B) suggest otherwise. It will soon emerge that (A) and (B) are immediate implications of our best theory of vagueness: precise supervaluationism.

We shall proceed as follows. $\$ 1$ formulates representationalism. $\mathbf{2}$ discusses the basics of vagueness, and introduces precise supervaluationism. $\$ 3$ argues that precise supervaluationism implies (A) and (B). §4 proves (in a classical setting) that representationalism is inconsistent with $(\mathrm{A})$ and $(\mathrm{B})$, provided that certain plausible assumptions are granted. Finally, $\$ \mathbf{5}$ briefly surveys the central lines of response available to representationalists.

For rhetorical purposes, it is convenient to present this paper as 'an objection against representationalism'. Nevertheless, such rhetorical flourishes should not obscure our central goal: to explore several fascinating issues at the intersection of perception and vagueness. 


\section{$\S 1 \quad$ Representationalism}

What is representationalism?

Begin with the following Harman-esque insight: perceiving the world involves perceptually predicating properties of objects in one's environment. ${ }^{1}$ For example: Avril perceptually predicates redness (or some specific shade of redness) of a tomato; Hal perceptually predicates sphericality (or some nearby shape) of a basketball. As these remarks indicate, we regard the locution ' $x$ perceptually predicates $y$ ' (where $x$ is a perceiver and $y$ is a property) as the distinctive theoretical ideology of representationalism. We shall also employ locutions of the form ' $x$ perceptually predicates $y$ of $z_{1} \ldots z_{n}{ }^{\prime}$, where $z_{1} \ldots z_{n}$ are objects which $x$ perceives, and $y$ is an $n$-adic property. Such locutions are, of course, connected: if there exist objects $z_{1} \ldots z_{n}$ such that $x$ perceptually predicates $y$ of $z_{1} \ldots z_{n}$, then $x$ perceptually predicates $y$. However, the converse arguably fails: in cases of 'perfect hallucination', perceivers perceptually predicate properties without perceptually predicating them of anything (Johnston 2004). For convenience, we occasionally shorten 'perceptual predication' to 'predication'.

According to representationalism, there is an intimate connection between phenomenal consciousness and perceptual predication.

\section{Representationalism}

For every phenomenal property $x$, there is some property $y$ such that: a subject instantiates $x$ iff she perceptually predicates $y{ }^{2}$

Formally: $\forall x$ (phenomenal $(x) \rightarrow \exists y \forall z(z$ instantiates $x \leftrightarrow z$ predicates $y$ )

In general, the predicable property associated with a given phenomenal property will be extremely complex, encoding a wealth of information about the world.

Representationalism is the most popular account of perceptual phenomenology in contemporary philosophy. ${ }^{3}$ Some representationalists (e.g. Tye

\footnotetext{
${ }^{1}$ See Harman (1990). The notion of perceptual predication is discussed by many writers. Among the best recent treatments are Siegel (2010) and Pautz (2009). ${ }^{2}$ This is a strict biconditional: necessarily, a subject instantiates $x$ iff she perceptually predicates $y$.
} 
1995 and Dretske 1995) are motivated by reductive ambitions, driven by the hope that consciousness can be naturalized through a causal-covariational account of perceptual predication. Others (e.g. Pautz 2006) have no reductive ambitions whatever, and simply regard representationalism as an interesting thesis about the relationship between phenomenal consciousness and perceptual predication. We suspect that most proponents of 'naïve realism' also subscribe to representationalism (when formulated as above), even though naïve realism is often construed as an alternative to representationalism. ${ }^{4}$

Three notes on our formulation of representationalism. First: we do not propose to define 'phenomenal property' in this paper, and assume that readers are familiar with this now-ubiquitous notion (Nagel 1974; Block 1995). As our formulation of representationalism indicates, we assume that subjects are bearers of phenomenal properties; two subjects are phenomenal duplicates iff they have exactly the same phenomenal properties. (Some philosophers prefer to treat experiences as bearers of phenomenal properties, but any differences between these approaches are almost certain to be merely verbal.)

Second: when formalizing representationalism, we have elected to employ a first-order language enriched with the predicate ' $x$ instantiates $y$ '. We thereby assume that nominalism about properties is false. It may be possible to formulate representationalism in a higher-order language acceptable to nominalists which contains no ‘instantiation' predicate (cf. Williamson 2004). However, higher-order languages raise difficult complications which we prefer to sidestep here.

Finally: there are, of course, alternative formulations of representationalism. Some of these alternatives matter in the present context whereas others do not. One version of representationalism that isn't targeted by the argument developed in this paper is what one might call weak representationalism:

\section{Weak Representationalism}

For any phenomenal property $x$, some property $y$ is such that: a subject instantiates $x$ if she perceptually predicates $y$.

\footnotetext{
${ }^{3}$ Proponents of representationalism (or something near enough) include Byrne (2001), Dretske (1995), Johnston (2004), Harman (1990), Hill (2009), Pautz (2007) and Tye $(1995 ; 2000)$.

${ }^{4}$ For more on this theme, see Siegel (2010).
} 
The argument developed in this paper does not target weak representationalism because weak representationalists are committed only to one direction of the biconditional implied by full-strength representationalism. (The 'if' direction, not the 'only if' direction.) We return to this issue in $\$ \mathbf{4}$.

Other versions of representationalism are modality-specific insofar as their scope is restricted to perceptual phenomenology in a particular sensory modality. Consider, for example, the following version of representationalism:

\section{Visual Representationalism}

For any visual phenomenal property $x$, some property $y$ is such that: a subject instantiates $x$ iff she visually predicates $y$.

Visual representationalism exchanges the general notion of perceptual predication for the more specific notion of visual predication, and the general notion of a phenomenal property for the more specific notion of a visual phenomenal property. Every argument provided below applies mutatis mutandis to visual representationalism.

Finally, there are versions of representationalism that eschew talk of properties for talk of propositions. Consider, for example, the following claim:

\section{Propositional Representationalism}

For any phenomenal property $x$, some proposition $p$ is such that: a subject instantiates $x$ iff she perceptually represents $p$.

This version of representationalism is also targeted by the argument of this paper, for it is plausible that propositional representationalism is equivalent to our formulation of representationalism. Here is why. Let $C$ be a phenomenal property. For heuristic purposes, assume that $C$ is a phenomenal property which ordinary subjects instantiate when confronted with a red square and a blue circle. According to propositional representationalism, a subject instantiates $C$ iff she perceptually represents the following proposition (or something near enough):

\section{$\exists x \exists y(\operatorname{red}(x) \& \operatorname{square}(x) \& \operatorname{blue}(y) \& \operatorname{circular}(y))$}


As Pautz (2007) observes, presumably a subject perceptually represents the foregoing proposition iff she perceptually predicates the following complex property:

$$
\lambda x \lambda y(\operatorname{red}(x) \& \operatorname{square}(x) \& \operatorname{blue}(y) \& \operatorname{circular}(y))
$$

Informally, the expression ' $\lambda x \lambda y(\operatorname{red}(x) \& \operatorname{square}(x) \&$ blue $(y) \&$ circular $(y))$ ' expresses a complex dyadic property which an object $x$ and an object $y$ instantiate iff $x$ is a red square and $y$ is a blue circle. Using $\lambda$-abstraction, ${ }^{5}$ it is always possible to construct a complex property which corresponds to any given proposition in the manner described above. For this reason, it is plausible that propositional representationalism implies our formulation of representationalism.

\section{$\S 2 \quad$ Vagueness and Precise Supervaluationism}

The remainder of this paper argues that representationalism conflicts with a popular (and plausible) account of vagueness: precise supervaluationism. This section begins by discussing the basics of vagueness before turning to the central claims of precise supervaluationism.

The concept of vagueness is best introduced by appeal to the concept of a borderline case. In this connection, Cian Dorr writes:

The concept [of a borderline case] has its most basic application when we are faced with a question of the form 'Is $x F$ ?', but are unwilling to answer 'yes' or 'no' for a certain kind of distinctive reason. Wanting to be co-operative, we need to say something; by saying 'it's a borderline case', we excuse our failure to give a straightforward answer while conveying some information likely to be of interest to the questioner. ${ }^{6}$

Examples are plenitudinous. Is Hal bald? It's a borderline case. Is Avril old? It's a borderline case. Is a particular patch of colour an instance of red or of orange? It's a

\footnotetext{
${ }^{5}$ See Sider (2010) for an introduction to $\lambda$-abstraction.

${ }^{6}$ Dorr 2010: 550.
} 
borderline case. Is Orin tall? It's a borderline case. Such 'borderline case'-talk is commonly regimented using the one-place sentential operator 'it is vague whether...' Thus, we say:

1. It is vague whether Hal is bald.

2. It is vague whether Avril is old.

3. It is vague whether Orin is tall.

In formal languages, the operator 'it is vague whether...' is customarily replaced with the less-cumbersome operator $\nabla .7$ Thus, 'it is vague whether Hal is bald' is rendered: $\nabla B h$.

The concept of vagueness is connected to the concept of determinacy. It is determinate that an ordinary man with 3 hairs on his head is bald. It is determinate that a three-foot grown woman is not tall. It is determinate that 5,000,000,000 grains of sand placed closely together compose a heap. It is determinate that scarlet is a shade of red. When theorizing in a formal language, the operator 'it is determinate that...' is customarily replaced with the less-cumbersome operator $\Delta$. Thus, 'it is determinate that Hal is bald' is rendered: $\Delta B h$. The following schemata capture the connections between vagueness and determinacy:

$$
\nabla \phi \leftrightarrow(\sim \Delta \phi \& \sim \Delta \sim \phi)
$$

Informally: it is vague whether $\phi$ iff (it is neither determinate that $\phi$, nor determinate that not- $\phi)$.

$$
\Delta \phi \leftrightarrow(\phi \& \sim \nabla \phi)
$$

Informally: it is determinate that $\phi$ iff ( $\phi$ and it is not vague whether $\phi)$. If you understood vagueness, you now understand determinacy.

Before we proceed further, two notes on logical matters. First, we will employ classical propositional and first-order logic. Although some philosophers deny that classical logic has any useful application to vague languages, we will ignore such

\footnotetext{
${ }^{7}$ See Williamson (1999), among many others.
} 
concerns here, and adopt the fairly widespread assumption that classical logic provides an extremely valuable framework for reasoning about vagueness - one that cannot easily be replaced. ${ }^{8}$

Second, we shall assume that every instance of the following schema holds:

$$
\left(\mathrm{K}_{\Delta}\right) \quad \Delta(\phi \rightarrow \psi) \rightarrow(\Delta \phi \rightarrow \Delta \psi)
$$

Informally: if a conditional is determinate, and its antecedent is also determinate, then so is its consequent. $\left(\mathrm{K}_{\Delta}\right)$ corresponds to a familiar schema for the necessity operator $\square:$

$\left(\mathrm{K}_{\square}\right) \quad \square(\phi \rightarrow \psi) \rightarrow(\square \phi \rightarrow \square \psi)$

Informally: if a conditional is necessary, and its antecedent is also necessary, then so is its consequent. It is extremely commonplace to use $\left(\mathrm{K}_{\Delta}\right)$ and $\left(\mathrm{K}_{\square}\right)$ when reasoning with $\Delta$ and $\square$ (cf. Williamson 1999). In the absence of such schemata, it is impossible to prove anything interesting about either necessity (/possibility) or determinacy (/vagueness).

So much for the basics. We now turn to precise supervaluationism, the most popular account of vagueness in contemporary philosophy. Let us begin with a simple example. It is vague whether 20 is a small number. (If you don't agree that 20 is a small number, choose some other number. The predicate ' $x$ is a small number' is extremely context-sensitive, so there is nothing wrong with assuming that we are in a context in which it is vague whether 20 counts as small.) For heuristic purposes, let us also assume that hardcore Platonism is true: some object is determinately identical to the number 20 .

On one view - the Unpopular View - there is a particular property $V$ which the predicate ' $x$ is a small number' determinately expresses. It is vague whether 20 is a small number because it is vague whether 20 has $V$. In the lingo, $V$ is a 'vague property'. Picture the matter thus:

\footnotetext{
${ }^{8}$ As Robbie Williams observes in a recent draft (ms: 1), 'classical treatments of indeterminacy are on the march. A growing number of authors argue that we can have an adequate theory of indeterminacy or vagueness that demands no revision of the classicism-presupposing theories used throughout the sciences.'
} 


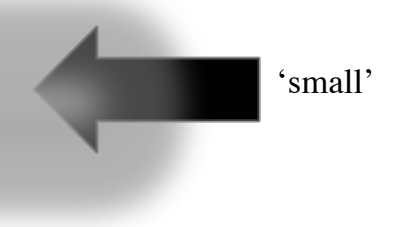

Figure 1: $V$ is represented by a fuzzy grey blob. The sharp black arrow indicates that 'small' determinately expresses $V$.

Many philosophers are deeply suspicious of the view depicted by Figure 1. These philosophers hold that 'the world itself' is wholly precise, even though language is ridden with imprecision. On this view, vague properties do not exist: there is no particular property which the predicate ' $x$ is a small number' determinately expresses. Rather, there are many precise properties in the vicinity, and it is vague which of them is expressed by the predicate ' $x$ is a small number':

the property of being less than 18 ;

the property of being less than 19;

the property of being less than 20;

the property of being less than 21 ;

etc.

Let us dub these properties small-candidates. Notice that every small-candidate is completely precise: every number is either determinately less than 18 or determinately not less than 18 , determinately less than 19 or determinately not less than 19 , and so forth. Picture the matter thus: 


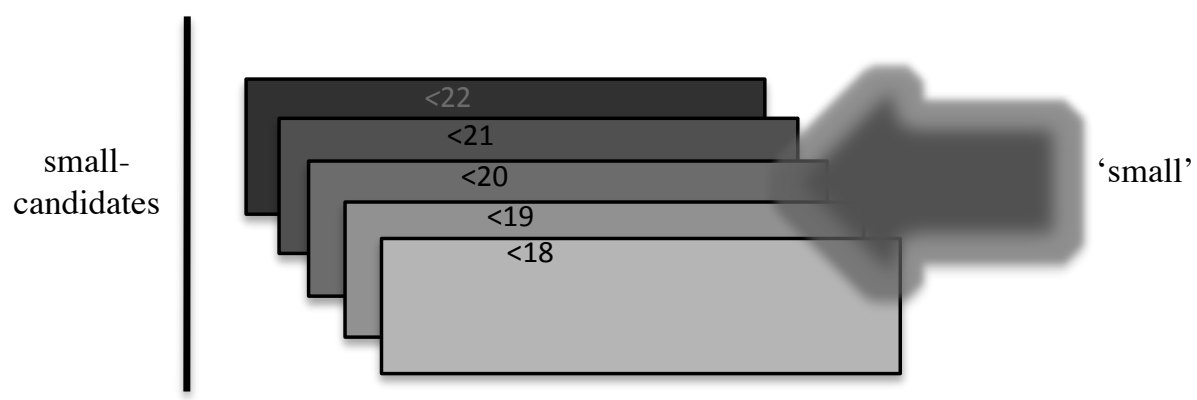

Figure 2: The rectangles represent small-candidates (the property of being less than 18 , 19, etc). The fuzzy arrow indicates that it is vague which small-candidate is expressed by 'small'.

Of course, not every property is a small-candidate: the property of being greater than 1 is not a small-candidate; nor (in most contexts) is the property of being less than $10^{13}$. Crucially, it is vague whether 20 is a small number iff 20 has some (but not all) small-candidates. Thus, 20 has the property of being less than 21 , but does not have the property of being less than 18 . Since these properties are both small-candidates, it follows that it is vague whether 20 is a small number.

As noted above, every small-candidate is precise. There is no fuzzy grey blob in the ontology depicted by Figure 2; every property is a crisp rectangle. Accordingly, the following inference is invalid:

It is vague whether $\alpha$ is $F$.

Therefore, there is some property $V$ such that it is vague whether $\alpha$ instantiates $V$.

From the fact that it is vague whether $\alpha$ is $F$, it follows merely that there are various $F$-candidates - some of which $\alpha$ determinately has, and the rest of which $\alpha$ determinately does not have. It does not follow that there is some 'vague property' $V$ such that it is vague whether $\alpha$ instantiates $V$.

That, in a nutshell, is the basic idea behind precise supervaluationism. The same idea applies across all cases of vagueness. 'Bald-candidates' are precise properties pertaining to the distribution of hair. It is vague whether Tom is bald because Tom has some (but not all) bald-candidates. 'Red-candidates' are precise properties which correspond to precise regions of colour space. It is vague whether a given shade is red because it has some (but not all) red-candidates. And so forth. Let 
us provide a more rigorous (and general) formulation of precise supervaluationism. We begin with the following definitions:

For any property $x$ and any predicate $F: x$ is an $F$-candidate iff either $F$ determinately expresses $x$, or it is vague whether $F$ expresses $x$. Formally: $\Delta(F$ expresses $x) \vee \nabla(F$ expresses $x)$.

When there is more than one $F$-candidate, we say that it is vague which property $F$ expresses.

A property $x$ is precise iff necessarily, there is no object $y$ such that it is vague whether $y$ instantiates $x$. Formally: $\square \sim \exists y \nabla(y$ instantiates $x)$. Otherwise, $x$ is vague.

Precise supervaluationism comprises two claims.

\section{Precise Supervaluationism}

(i) Every property is precise.

(ii) For any monadic predicate $F$ and any object $x$ : it is vague whether $F$ applies to $x$ iff $x$ instantiates some (but not all) $F$-candidates. ${ }^{9}$

The idea, then, is that every vague predicate $F$ is associated with a variety of precise properties or 'candidates' $y_{1} \ldots y_{n}$ such that it is vague which $y_{i}$ is expressed by $F$. Vagueness arises when an object has some (but not all) $y_{i}$. As we observed above, it is vague whether $\phi$ iff it is neither determinate that $\phi$, nor determinate that not- $\phi$. Thus, precise supervaluationism implies that for any monadic predicate $F$ and any object $x$, it is determinate that $F$ applies to $x$ iff $x$ instantiates every $F$-candidate. The number 0 , for example, is determinately small, since it instantiates every small-candidate.

It is important to stress that the vast majority of precise supervaluationists accept the following claim:

\footnotetext{
${ }^{9}$ Likewise for polyadic predicates: for any objects $x_{1} \ldots x_{n}$, it is vague whether an $n$ place predicate $F$ applies to an $n$-tuple $\left\langle x_{1} \ldots x_{n}\right\rangle$ iff $\left\langle x_{1} \ldots x_{n}\right\rangle$ is in the extension of some (but not all) $F$-candidates.
} 
(1) Some property is expressed by 'small'.

$\exists x(x$ is expressed by 'small')

Precise supervaluationists merely hold that it is vague which property is expressed by the relevant word. Thus, they deny the following de re claim:

(2) Some property is determinately expressed by 'small'.

$\exists x \Delta(x$ is expressed by 'small')

By accommodating vagueness without admitting vague properties, precise supervaluationism captures the familiar suggestion that 'the world itself' does not suffer from vagueness. Vagueness arises only when our representations fail to determinately latch onto any particular chunk of the world. Thus, Michael Dummett writes:

The notion that things might actually be vague, as well as being vaguely described, is not properly intelligible..$^{10}$

Likewise, Bertrand Russell writes:

There is a certain tendency in those who have realized that words are vague to infer that things are also vague... This seems to me precisely a case of the fallacy of verbalism - the fallacy that consists in mistaking the properties of words for the properties of things. Vagueness and precision alike are characteristics which can only belong to a representation $[\ldots]^{11}$

In the same vein, David Lewis writes:

The only intelligible account of vagueness locates it in our thought and language. The reason it's vague where the outback begins is not that there's this thing, the outback, with imprecise borders. Rather, there are many things,

\footnotetext{
${ }^{10}$ Dummett 1975: 260.

${ }^{11}$ Russell 1923: 83.
} 
with different borders, and nobody has been fool enough to try to enforce a choice of one of them as the official referent of the word 'outback'. Vagueness is semantic indecision. ${ }^{12}$

Other proponents of precise supervaluationism include Kit Fine (1975), Vann McGee and Brian McLaughlin (1995), Kirk Ludwig and Greg Ray (2002), David Braun and Ted Sider (2007), Timothy Williamson (2003), and many, many others. As Williamson himself has pointed out, precise supervaluationism is compatible with (and perhaps even entailed by) certain versions of epistemicism: see Williamson (ibid).

Before we move on, two brief observations. First, we do not assume that $\ulcorner\phi\urcorner$ is true only if it is determinate that $\phi$, as some (but certainly not all) supervaluationists have claimed. ${ }^{13}$ Second, the version of supervaluationism developed in this chapter falls far short of providing a complete supervaluationist semantic theory for a vague first-order language. In our judgment, however, there is little to be gained from expounding the minutiae of such a theory; readers are instead advised to consult the works cited above.

We have not provided any positive argument for precise supervaluationism; nor have we surveyed its alternatives. Instead, we shall simply assume that precise supervaluationism is correct. Given the status of precise supervaluationism in the literature, we think that this stance is not unjustified: even foes of precise supervaluationism should be interested in its ramifications on the metaphysics of perception.

\section{$\$ 3 \quad$ Precise Supervaluationism and Perception}

This section argues that precise supervaluationism implies the following claims:
A. Perceptual representation is vague.
B. Phenomenal consciousness is precise.

\footnotetext{
${ }^{12}$ Lewis 1986: 212-13.

${ }^{13}$ This claim (sometimes expressed as the view that 'truth = supertruth') has become increasingly unpopular since McGee and McLaughlin's classic 1995 paper.
} 
To keep things simple, let us focus on a particular perceiver-call her 'Avril'. (In what follows, 'Avril' is treated as a free variable standing for an arbitrary perceiver.) Avril, we may suppose, is viewing a medium-sized canvas (call it ' $\beta$ '), painted a uniform shade of dark red. $\beta$ is located one foot in front of Avril, but lies in the periphery of her visual field. For simplicity, we shall assume that Avril does not perceive any other object. This assumption can easily be eliminated, but it enables us to avoid needless epicycles. Avril is an ordinary perceiver, viewing $\beta$ under normal conditions. Presumably, therefore, it is determinate that she perceives $\beta$ accurately.

Nevertheless, it could easily have been vague whether Avril perceives $\beta$ accurately. To see that this is so, suppose that the superdeterminate shade of dark red that $\beta$ actually has is $\mathrm{R}_{500}$. Let $<w_{@}, w_{1}, \ldots, w_{k}>$ be a finite sequence of possible worlds, where $w_{@}$ is the actual world. $\beta$ has very slightly different shades of colour at every world in the sequence. Thus, $\beta$ has $\mathrm{R}_{500}$ in $w_{@}$. $\beta$ has $\mathrm{R}_{499}$ in $w_{1} \cdot \beta$ has $\mathrm{R}_{498}$ in $w_{2}$. $\beta$ has $\mathrm{R}_{497}$ in $w_{3}$. And so forth. In $w_{k}$-the final world in the sequence- $\beta$ is no longer dark red, but some maximally specific shade of bright orange. However, let us stipulate that Avril perceives $\beta$ in exactly the same way at every world in the sequence. That is: she perceptually attributes exactly the same properties to $\beta$, and has exactly the same phenomenal properties, in every world. The canvas changes colour from world to world; Avril's perception of the canvas remains constant.

It should be clear that $\left\langle w_{@}, w_{1}, \ldots, w_{k}>\right.$ forms a Sorites sequence. It is determinate that Avril perceives $\beta$ accurately in $w_{@}$. Her perceptual system is functioning normally, and she is viewing $\beta$ under ordinary conditions. Likewise, it is determinate that Avril does not perceive $\beta$ accurately in $w_{k}$ : $\beta$ is bright orange in $w_{k}$, yet Avril continues to perceptually attribute some shade of red to $\beta$. Nevertheless, there are many worlds $w_{i}$, positioned near the middle of the Sorites sequence, such that it is vague whether Avril perceives $\beta$ accurately in $w_{i}$. We suspect that many readers will (correctly) regard this claim as a banal truth. Not convinced? Notice that if it is not vague whether Avril perceives $\beta$ accurately at any world in the sequence, it immediately follows that:

(1) For some world $w_{i}$ : it is determinate that Avril perceives $\beta$ accurately in $w_{i}$, and it is determinate that Avril does not perceive $\beta$ accurately in $w_{i+1}$. 
Surely there is no such 'determinate cut-off point' in the series of worlds $<w_{@}, w_{1}, \ldots, w_{k}>$, any more than there is a determinate cut-off point separating small numbers from large numbers. There must, therefore, be various worlds $w_{i}$ such that it is vague whether Avril perceives $\beta$ accurately in $w_{i}$, just as there are various numbers $n$ such that it is vague whether $n$ is small. ${ }^{14}$

$\beta$ changes colour across $\left\langle w_{@}, w_{1}, \ldots, w_{k}\right\rangle$. However, there is nothing special about colour; we can set up the Sorites series in various alternative ways and arrive at the same result. For example, let us stipulate that $\beta$ changes its location across $<w_{@}$, $w_{1}, \ldots, w_{k}>$, at a rate of $0.001 \mathrm{~mm}$ per world. By the end of the sequence, $\beta$ has moved an entire metre away. Throughout the series, Avril's perception of $\beta$ is held fixed. It is determinate that Avril perceives $\beta$ accurately in $w_{@}$. It is determinate that Avril does not perceive $\beta$ accurately in $w_{k}$. But for many worlds $w_{i}$, positioned near the middle of the Sorites sequence, it is vague whether Avril perceives $\beta$ accurately in $w_{i}$.

Henceforth, let us adopt the assumption that we occupy one of the worlds $w_{i}$ in which it is vague whether Avril perceives $\beta$ accurately. We pose the following question: what follows from that fact that it is vague whether Avril perceives $\beta$ accurately?

On one view, it follows that there is some vague colour property $V$ which Avril determinately predicates of $\beta$. It is vague whether Avril perceives $\beta$ accurately because it is vague whether $\beta$ has $V$. Of course, this suggestion is wholly antithetical to precise supervaluationism. There are no vague properties in the precise supervaluationist's ontology. How, then, should the proponent of precise supervaluationism explain the fact that it is vague whether Avril perceives $\beta$ accurately?

To answer this question, let us return to the simpler case of vague predicates such as ' $x$ is a small number', discussed above. According to precise supervaluationism, various precise properties are small-candidates; it is vague which of them is expressed by the predicate ' $x$ is a small number'. Furthermore, it is vague whether the latter predicate applies to the number 20 because 20 has some (but not all) small-candidates. This account can be straightforwardly extended to the case of vague perceptual accuracy, along the following lines:

\footnotetext{
${ }^{14} \mathrm{We}$ stress that this assertion is compatible with any sensible theory of vagueness, including epistemicism (Williamson 1994) and other classical-logic-endorsing views.
} 
(i) Say that a property is 'relevant' iff it is a colour property, or a conjunctive property including some colour property as a conjunct.

(ii) There is no relevant property $V$ such that Avril determinately predicates $V$-just as there is no particular property $V$ such that ' $x$ is a small number' determinately expresses $V$. Formally: $\sim \exists x(\operatorname{relevant}(x) \& \Delta$ (Avril predicates $x$ )).

(iii) Rather, there are various colour properties in the vicinity, and it is vague which of them Avril predicates - just as there are various precise arithmetical properties in the vicinity, and it is vague which of them is expressed by ' $x$ is a small number'. Formally: $\exists y_{1} \ldots \exists y_{n}\left(\nabla\right.$ (Avril predicates $\left.y_{1}\right)$ $\& \ldots \nabla\left(\right.$ Avril predicates $\left.\left.y_{n}\right)\right)$.

(iv) If it is vague whether Avril predicates $y$, then $y$ is a 'contentcandidate'. The content-candidates comprise certain precise colour properties (corresponding to precise regions of colour space), ${ }^{15}$ and conjunctive properties containing such colours as conjuncts.

(v) It is vague whether ' $x$ is a small number' applies to the number 20 because 20 has some (but not all) small-candidates. Likewise, it is vague whether Avril perceives $\beta$ accurately because $\beta$ has some (but not all) contentcandidates.

It seems to us that precise supervaluationists are required to adopt this account. In any case, we cannot readily envisage any alternatives. Thus, every precise supervaluationist is committed to assumption (A):

\footnotetext{
${ }^{15}$ It is important to stress that content-candidates need not be superdeterminate colours. Content-candidates may also be determinable colours. The crucial point is that each content-candidate $C$ is precise: there is no object $x$ such that it is vague whether $x$ has $C$. The notion of a 'precise determinable property' is perfectly coherent.
} 


\section{A. Perceptual representation is vague}

There is no relevant property which Avril determinately predicates.

$\sim \exists x(\operatorname{relevant}(x) \& \Delta($ Avril predicates $x))$

That is the first implication of precise supervaluationism on the metaphysics of perception, and it is by no means a trivial one. Precise supervaluationism has a second important implication:

\section{B. Phenomenal consciousness is precise}

For all phenomenal properties $x$ : it is not vague whether Avril instantiates $x$. $\forall x(\operatorname{phenomenal}(x) \rightarrow \sim \nabla($ Avril instantiates $x))$

After all, precise supervaluationists accept the following universal generalization: $\forall x \forall y \sim \nabla(x$ instantiates $y)$. As noted above, 'Avril' is a free variable, standing for an arbitrary perceiver. Therefore, it is permissible to instantiate 'Avril' in the latter generalization to obtain: $\forall x \sim \nabla($ Avril instantiates $x)$ ). This trivially implies (B). ${ }^{16}$

\section{$\S 4$ The Proof}

(A) and (B) generate serious problems for representationalism. The difficulty should be pre-theoretically apparent: representationalism claims that there is an extremely close connection between phenomenal consciousness and perceptual representation; (A) and (B) suggest otherwise. According to (A), perceptual representation is a vague matter; according to (B), phenomenal consciousness is not. Indeed, provided that we make one prima facie plausible assumption, (A) and (B) are provably inconsistent with representationalism.

Consider the following claims:

\footnotetext{
${ }^{16}$ Why have we continually reminded the reader that 'Avril' is a free variable standing for an arbitrary object? It is widely recognized that the following inference is valid, where $\alpha$ is a free variable standing for an arbitrary object: ' $\forall x \Delta \phi(x)$; therefore, $\Delta \phi(\alpha)^{\prime}$. However, it is equally widely recognized that same inference is invalid if $\alpha$ is a vague noun phrase (cf. Lewis 1988; Williamson 2003; Williams 2008). It is important, therefore, to stress that 'Avril' is a variable standing for an arbitrary object, not a vague proper name.
} 


\section{Representationalism}

$\forall x$ (phenomenal $(x) \rightarrow \exists y \forall z(z$ instantiates $x \leftrightarrow z$ predicates $y)$

For every phenomenal property $x$, there is some property $Y$ such that:

a subject $z$ instantiates $x$ iff $z$ predicates $y$.

\section{$\Delta$-Representationalism}

$\forall x$ (phenomenal $(x) \rightarrow \exists y \forall z \Delta(z$ instantiates $x \leftrightarrow z$ predicates $y)$

For every phenomenal property $x$, there is some property $y$ such that for any subject $z$ it is determinate that: $z$ instantiates $x$ iff $z$ predicates $y$.

The 'plausible assumption' required to prove the incompatibility of representationalism with (A) and (B) is this: philosophers who accept representationalism should also accept $\Delta$-representationalism. There is no doubt that this assumption is pre-theoretically attractive. Representationalism is a substantive thesis about the metaphysics of perception; if it is true, then surely its truth-value will survive the insertion of a well-placed determinacy operator. It is doubtful that many card-carrying representationalists will be happy to deny $\Delta$-representationalism. (Personal correspondence has confirmed this impression.) Although the matter deserves considerable attention, we propose simply to assume-largely due to limitations of space - that philosophers should not accept representationalism without also accepting $\Delta$-representationalism. Using the $\left(\mathrm{K}_{\Delta}\right)$-schema introduced in §2, together with basic classical logic, we shall prove that (A), (B) and $\Delta$ representationalism collectively lead to contradiction.

Let $F$ be the most specific phenomenal property which Avril instantiates. $\Delta$ representationalism entails that:

(1) $\quad \exists y \forall z \Delta(z$ instantiates $F \leftrightarrow z$ predicates $y)$

Informally: there is some property $y$ such that for every subject $z$, it is determinate that $z$ instantiates $F$ iff $z$ predicates $y$. Let $G$ be an arbitrary property, and suppose for reductio that:

(2) $\quad \forall z \Delta(z$ instantiates $F \leftrightarrow z$ predicates $G)$ 
Evidently, $G$ is a complex conjunctive property, containing a variety of properties as conjuncts. Furthermore, one of its conjuncts must surely be a colour property.

Otherwise it would be manifestly false that every subject who predicates $G$ instantiates $F$. Thus, $G$ is relevant (in the sense characterized in $\$ 3$ ):

(3) $\operatorname{relevant}(G)$

Since 'Avril' is a variable standing for an arbitrary perceiver, we can plug 'Avril' into (2) to obtain:

(4) $\Delta$ (Avril instantiates $F \leftrightarrow$ Avril predicates $G)$

By assumption,

(5) Avril instantiates $F$

(B) entails:

(6) $\quad \sim \nabla($ Avril instantiates $F)$

By the definition of $\Delta$ (introduced in $\S 2),(5)$ and (6) entail:

(7) $\Delta($ Avril instantiates $F)$

Applying the $\left(\mathrm{K}_{\Delta}\right)$-schema, (4) and (7) entail:

(8) $\Delta$ (Avril predicates $G)$

(8) and (3) entail:

(9) $\operatorname{relevant}(G) \& \Delta$ (Avril predicates $G)$ 
Since $G$ is a variable standing for an arbitrary property, we can employ $\exists$-introduction to obtain:

(10) $\exists x(\operatorname{relevant}(x) \& \Delta($ Avril predicates $x))$

According to (10), there is some relevant property which Avril determinately predicates. But this straightforwardly contradicts (A). Therefore, by reductio, $\Delta$ representationalism is false. If representationalism entails $\Delta$-representationalism, then representationalism is also false.

Let us bring this section to a close by commenting on the following feature of the argument. $\Delta$-representationalism is a biconditional claim. As such, it can be split into two components:

$$
\begin{aligned}
& \text { Left-to-Right } \\
& \forall x(\text { phenomenal }(x) \rightarrow \exists y \forall z \Delta(z \text { instantiates } x \rightarrow z \text { predicates } y) \\
& \text { Right-to-Left } \\
& \forall x(\text { phenomenal }(x) \rightarrow \exists y \forall z \Delta(z \text { predicates } y \rightarrow z \text { instantiates } x)
\end{aligned}
$$

The argument advanced above only targets the left-to-right direction of $\Delta$ representationalism. As inspection of the argument will reveal, it is the left-to-right direction which enables us to derive (4), leading inexorably to the conclusion above. We have said nothing to challenge the right-to-left direction of $\Delta$-representationalism. One reaction to the argument, therefore, is to abandon the left-to-right direction of $\Delta$ representationalism, but to preserve the right-to-left direction. We assume, however, that representationalists would prefer to hold onto the full-strength biconditional.

\section{$\$ 5$ Conclusion}

One of the most common strategies for arguing against representationalism involves playing the counterexample game. The basic goal of the game is to produce a plausible case in which two subjects have the same phenomenal property, but predicate different properties (or vice versa). The argument developed in this paper is 
of an entirely different breed. It purports to provide a nonconstructive proof that representationalism is false. No concrete counterexample to representationalism has been produced; we have merely argued that representationalism is false on abstract and general grounds pertaining to the nature of vagueness, together with relatively uncontroversial logic.

How should representationalists respond? There are two main options. The first option is to deny precise supervaluationism, and embrace the existence of vague properties (see \$3). If vague properties exist, then the argument developed in $\$ 4$ fails. We no longer have any reason to accept premise (A) - the claim that there is no relevant property which Avril determinately predicates. To the contrary: if vague properties exist, then surely there is a relevant property $V$ which Avril determinately predicates. $V$ is a vague colour property; it is vague whether Avril perceives $\beta$ accurately because it is vague whether $\beta$ has $V$. Likewise, there is no longer any reason to accept premise (B) - the claim that it is never vague whether a subject has a given phenomenal property. This premise was motivated entirely by the view that vague properties do not exist. (Nevertheless, it would perhaps be surprising to discover that the viability of representationalism turns on the fate of controversial, substantive views about vagueness - though stranger things have happened.)

The second option is to accept representationalism, but deny $\Delta$ representationalism (see $\S 4$ ). On this view, representationalism is true, but not fully determinate. Assuming classical logic, this response renders precise supervaluationism entirely consistent with representationalism. (However, it also renders representationalism a deeply mysterious view.)

Although one of the present authors has argued against both responses at length in other work (Perkins ms), we won't pursue these matters here. Our overarching goal here has not been to develop a self-contained 'refutation' of representationalism, but to draw attention to a deep tension between two highlyplausible views, one in the philosophy of vagueness and the other in the philosophy of perception. Just how that tension might best be resolved is a matter that merits further investigation. ${ }^{17}$

\footnotetext{
${ }^{17}$ For countless discussions, thanks to John Hawthorne and especially Jeremy Goodman.
} 


\section{References}

Block, N. (1995) 'On a Confusion About a Function of Consciousness'. Behavioural and Brain Sciences 18: 227-47.

Braun, D. and Sider, T. (2007) 'Vague, So Untrue'. Nous 41: 133-156.

Byrne, A. (2001) 'Intentionalism Defended'. The Philosophical Review 110: 199-240.

Dorr, C. (2010) 'Iterating Definiteness'. In Cuts and Clouds, edited by Dietz and Moruzzi (Oxford University Press).

Dretske, F. (1995) Naturalizing the Mind. Oxford University Press.

Dummett, M. (1975) 'Wang's Paradox'. Synthese 30: 301-24.

Fine, K. (1975) 'Vagueness, Truth and Logic'. Synthese 30: 265-300.

Harman, G. (1990) 'The Intrinsic Quality of Experience'. Philosophical Perspectives 4: $31-52$.

Hill, C. (2009) Consciousness. Cambridge University Press.

Johnston, M. (2004) 'The Obscure Object of Hallucination'. Philosophical Studies 120: 113-183.

Lewis, D. (1986) On the Plurality of Worlds. Blackwell.

Lewis, D. (1988) 'Vague Identity: Evans Misunderstood'. Analysis 48: 128-30.

Ludwig, K. \& Ray, G. (2002) 'Vagueness and the Sorites Paradox'. Philosophical 
Perspectives 16: 419-461.

McGee, V. and McLaughlin, B. (1995) 'Distinctions Without A Difference'. Southern Journal of Philosophy 33 (Supp.): 203-251.

Nagel, T. (1974) 'What is it Like to be a Bat?' Philosophical Review 83: 435-456.

Pautz, A. (2006) 'Sensory Awareness is not a Wide Physical Relation’. Nous 40: 205240.

Pautz, A. (2007) 'Intentionalism and Perceptual Presence'. Philosophical Perspectives 21: 495-541.

Pautz, A. (2009) 'What are the Contents of Experiences?' The Philosophical Quarterly 59: 483-507.

Perkins, R. (ms) Vagueness and the Philosophy of Perception. BPhil Dissertation, University of Oxford.

Russell, B. (1923) 'Vagueness'. The Australian Journal of Philosophy and Psychology 1: 84-92.

Sider, T. (2010) Logic for Philosophy. Oxford University Press.

Siegel, S. (2010) 'Do Visual Experiences Have Content?' In Perceiving the World, edited by Nanay (Oxford University Press).

Tye, M. (1995) Ten Problems of Consciousness. MIT Press.

Tye, M. (2000) Consciousness, Color and Content. MIT Press.

Williams, J.R.G. (ms) ‘Classical Indeterminacy’. 
Williams, J.R.G. (2008) 'Multiple Actualities and Ontically Vague Identity'.

Philosophical Quarterly 58: 135-154.

Williamson, T. (1994) Vagueness. Routledge.

Williamson, T. (1999) 'On the Stucture of Higher-Order Vagueness'. Mind 108: 127143.

Williamson, T. (2003) 'Vagueness in Reality'. In The Oxford Handbook of Metaphysics, edited by Loux and Zimmerman (Oxford University Press).

Williamson, T. (2004) 'Everything’. Philosophical Perspectives 17: 415-465. 